\title{
Paths of Trains with Two-Wheeled Cars*
}

\author{
Luis Montejano $^{1,2}$ and Jorge Urrutia ${ }^{1}$ \\ ${ }^{1}$ Instituto de Matemáticas, Universidad Nacionál Autónoma de México, \\ México D.F., México \\ ${ }^{2}$ Facultad de Matemáticas, Universidad Autonoma de Guerrero, \\ Acapulco, Mexico
}

\begin{abstract}
In this paper we study the following simple and mind-puzzling problem: Can a model train car, which runs along an intricate track, complete a full cycle around it? In our paper a track will be represented by a simple closed curve, and the cars of our model train by segments whose endpoints lie on the curve.
\end{abstract}

\section{Introduction}

Let $C$ be a simple closed curve in the plane that can be thought of as a track. Imagine a model train car with a single wheel at each end, which we run along the track of $C$. We ask the following question. What lengths $\lambda$ may the car have that allow it to traverse all of $C$ ? If $C$ is a circle, any car whose length $\lambda$ is less than or equal to the diameter of $C$ will be able to run around the entire length of the track. If $C$ is an ellipse, any car with $\lambda$ greater than the length of its smallest axis will, however, get stuck.

Consider a train of $n$ such cars linked together, traveling along the curve $C$. Once again, it is interesting to ask what car lengths will allow the train to traverse the entire curve.

We encourage the reader to try experimenting with trains of a variety of car lengths running along different curves before proceeding to read the remainder of the article. It is an entertaining, instructive and sometimes surprising exercise to work out trajectories that will allow the train to complete a circuit of the entire curve. For example the reader may verify by himself that for the curve shown in Fig. 1, the car represented by the line segment with small circles at its endpoints can traverse the whole curve.

In [1] Goodman et al. studied similar problems but from a different perspective than the one we have. While they emphasis the discrete approach, we emphasis the topological

\footnotetext{
* Luis Montejano was supported by CONACYT 41340 and DGAPA IN111702, and Jorge Urrutia was supported by CONACYT of Mexico, Proyecto 37540-A.
} 


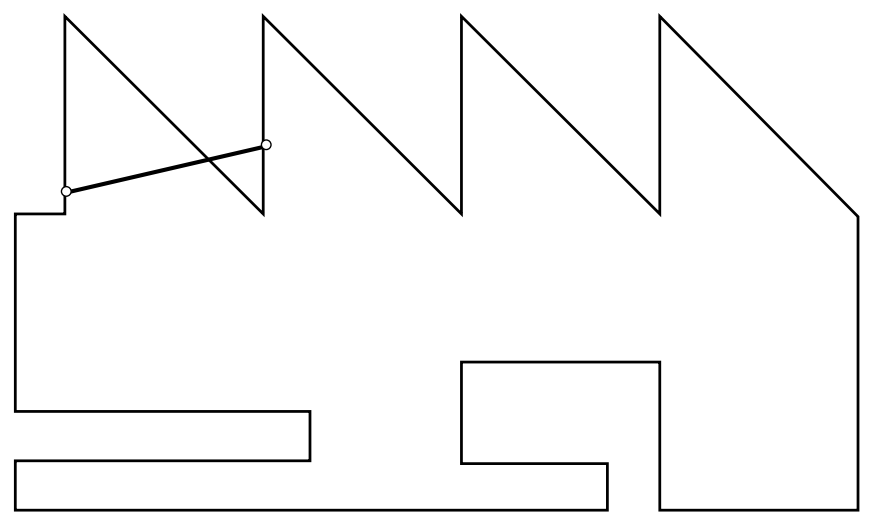

Fig. 1

approach. More precisely, our cars run along piecewise differential or piecewise analytic curves and our concept of "transverse the whole curve" is topological because we require the car to transverse the curve essentially once (degree 1), instead they require for the car only to pass through every point of the curve. So, our main problem is different and therefore our conclusions are also different.

Let $\alpha: S^{1} \rightarrow R^{2}$ be a parameterization of the simple closed curve $C$, where $S^{1}$ denotes the unit circle in $R^{2}$. We require here that $\alpha$ be an injective, piecewise differentiable (analytic) function. Consider the function

$$
\Lambda: S^{1} \times S^{1} \rightarrow R
$$

given by $\Lambda(x, y)=\|\alpha(x)-\alpha(y)\|$, for all $x, y \in S^{1} . \Lambda^{-1}(\lambda)$ is the space of all possible positions of cars of length $\lambda$ on curve $C$.

Definition 1. We say that a car of length $\lambda$ traverses along the curve $C$ if the following continuous function exists:

$$
\psi=\left(\psi_{1}, \psi_{2}\right): S^{1} \rightarrow \Lambda^{-1}(\lambda) \subset S^{1} \times S^{1},
$$

where $\psi_{1}, \psi_{2}: S^{1} \rightarrow S^{1}$ are the coordinate functions of $\psi$. If in addition $\psi_{1}: S^{1} \rightarrow S^{1}$ has degree \pm 1 , then we say that the car of length $\lambda$ traverses the entire curve $C$.

Informally speaking, we say that a car of length $\lambda$ can traverse entirely the curve $C$ if its back wheel traverses $C$ essentially once (degree 1). Since $\psi$ is a function defined on $S^{1}$, the initial and final positions of the car before and after the back wheel makes a complete transversal of $C$ must be the same.

\section{Questions About Car Paths}

Question 1. Can a car of length $\lambda$ traverse $C$ without repeating a position, but in such a way that its back wheel traverses $C$ essentially more than once? 
Question 2. Let $\psi: S^{1} \rightarrow \Lambda^{-1}(\lambda) \subset S^{1} \times S^{1}$ be a path by which a car of length $\lambda$ traverses the entire curve $C$, that is, a route in which the back wheel traverses $C$ essentially once. Is it true that the front wheel then also traverses the curve essentially once? More formally, is it true that if $\psi_{1}: S^{1} \rightarrow S^{1}$ has degree \pm 1 , then $\psi_{2}: S^{1} \rightarrow S^{1}$ also has degree \pm 1 ?

One possible reason why a car longer than the minor axis of an ellipse could get stuck and be unable to traverse the entire ellipse, is if, as it turns, its orientation would become parallel to the orientation of the minor axis of the ellipse. This is not, however, possible; motivating the next question.

Question 3. If a train traverses the curve $C$ entirely, is it true that the orientations of its cars describe a complete revolution?

Question 4. If a car of length $\lambda$ traverses the curve $C$ entirely, and $\lambda^{\prime}<\lambda$, then is there a car of length $\lambda^{\prime}$ which can traverse $C$ entirely?

Question 5. Is traversing the entire curve $C$ by a car of length $\lambda$ a local or a global problem? In other words, is it possible for a "nice subarc" of $C$ to exist that allows a car of length $\lambda$ to traverse all of $C$ (in which the definition of "nice" involves only properties of the subarc itself)?

The last question arises from a situation such as that shown in Fig. 2. Furthermore, this example suggests that an affirmative answer to Question 4 is unlikely.

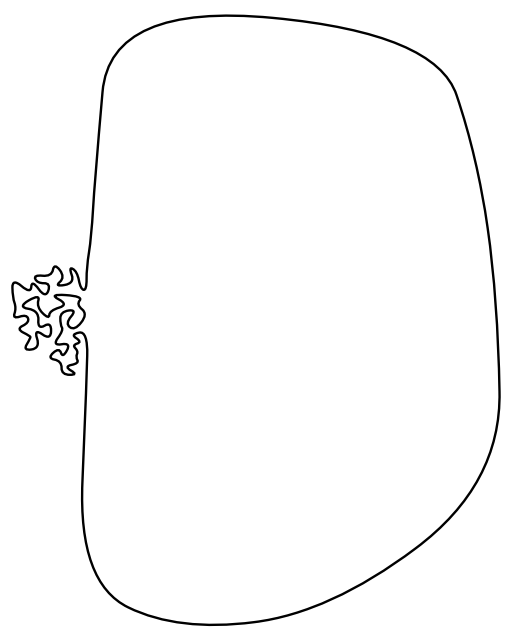

Fig. 2. In this figure, $C$ is essentially a circle in which a small portion of the circle has been replaced by a sector of a curve which can be as intricate as we might want. It is clear that a sufficiently long car can always traverse all of $C$. A small car might have problems getting out of the "intricate" sector of $C$. 


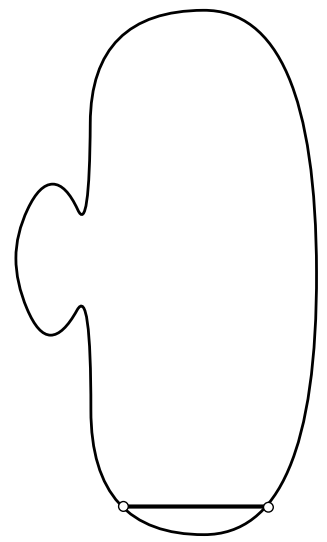

(a)

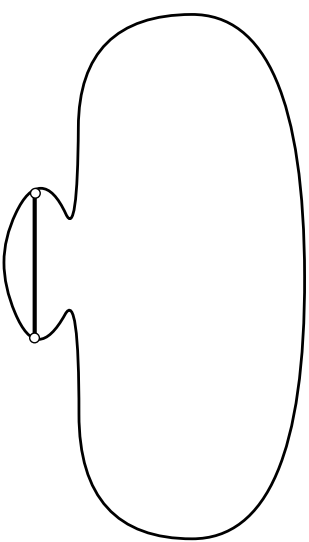

(b)

Fig. 3. The reader can easily verify that a car starting as shown in (a) can traverse the whole $C$, whereas in (b) it is stuck!

Our problem has aspects that make it more intriguing, as in some cases the starting position of the car determines if a car traverse all of $C$ or not. This situation is illustrated in Fig. 3.

\section{Answers to Section 2}

The first observation we make is that for $\lambda>0$,

$$
\Lambda^{-1}(\lambda) \cap \Delta=\emptyset
$$

where $\Delta=\left\{(x, x) \mid x \in S^{1}\right\}=\Lambda^{-1}(0)$ is the diagonal of $S^{1} \times S^{1}$.

A traversal of $C$ by cars of length $\lambda$ is thus a function $\phi=\left(\phi_{1}, \phi_{2}\right): S^{1} \rightarrow \Lambda^{-1}(\lambda) \subset$ $S^{1} \times S^{1}-\Delta$.

Functions of the unit circle $S^{1}$ on the torus $S^{1} \times S^{1}$ are classified homotopically by pairs of whole numbers. That is, a function $\phi$ has type $(n, m)$ if it wraps around the meridian of the torus $n$ times and $m$ times around its length. Two functions of the unit circle $S^{1}$ are homotopic on the torus if and only if they have the same type. Recall that the only curves of type $(n, m)$ which are not self-intersecting are those which have $n$ and $m$ relatively prime. Moreover, if the image of the function $\phi$ does not intersect the diagonal $\Delta$, then $\phi$ has type $(n, n)$ for some integer $n \in Z$. More details can be found in [3].

With this in mind, a traversal of $C$ by a car of length $\lambda$ which takes on distinct positions is an injective function $\phi: S^{1} \rightarrow \Lambda^{-1}(\lambda) \subset S^{1} \times S^{1}$ of type $\pm(n, n)$ with $n=0,1$. If $n=0$, the curve deforms to a point on the torus, and $\phi_{1}: S^{1} \rightarrow S^{1}$ is therefore of degree 0 . If $n=1$, then by definition, both $\phi_{1}: S^{1} \rightarrow S^{1}$ and $\phi_{2}: S^{1} \rightarrow S^{1}$ are of degree \pm 1 . This provides a negative answer to Question 1, an answer to Question 2, and allows us to formulate the characterization expressed in the following lemma. 
Lemma 1. The function $\psi=\left(\psi_{1}, \psi_{2}\right): S^{1} \rightarrow \Lambda^{-1}(\lambda) \subset S^{1} \times S^{1}$ represents a car of length $\lambda$ which traverses $C$ entirely if and only if $\psi: S^{1} \rightarrow \Lambda^{-1}(\lambda) \subset S^{1} \times S^{1}$ is a function of type $\pm(1,1)$; in other words, if and only if $\operatorname{deg}\left(\psi_{1}\right)=\operatorname{deg}\left(\psi_{2}\right)= \pm 1$.

To answer Question 3, let $\alpha$ be differentiable, and define the function

$$
\Theta: S^{1} \times S^{1} \rightarrow S^{1}
$$

as follows: $\Theta(x, y)=(\alpha(x)-\alpha(y)) /\|\alpha(x)-\alpha(y)\|$, if $x \neq y$ and $\Theta(x, x)=$ $\alpha^{\prime}(x) /\left\|\alpha^{\prime}(x)\right\|$. Note that $\Theta$ gives the tangent direction along $C$, which must be viewed as a point in $S^{1}$.

As $C$ is a simple closed curve, the successive directions of the tangents to $C$ will describe a complete revolution as $C$ is traversed completely. That is, the function $\Theta \delta: S^{1} \rightarrow S^{1}$ has degree \pm 1 where $\delta: S^{1} \rightarrow S^{1} \times S^{1}$ is the diagonal function $\delta(x)= \pm(x, x)$, for all $x \in S^{1}$. By Lemma $1, \psi: S^{1} \rightarrow \Lambda^{-1}(\lambda) \subset S^{1} \times S^{1}$ represents the path of a car of length $\lambda$ which traverses the entire curve if and only if $\psi$ is homotopic to $\delta$; therefore if and only if $\Theta \psi: S^{1} \rightarrow S^{1}$ has degree \pm 1 , that is, if and only if the orientations of the car makes topologically a complete revolution. Note that, by definition, the car goes back to its original position after it traverses the entire curve $C$. This gives rise to the following:

Theorem 1. A car of length $\lambda$ traverses the entire simple smooth curve $C$ if and only if the orientation of the car makes a complete revolution topologically.

We now answer Question 4 for piecewise analytic simple close curves. For example, polygonal curves are piecewise analytic. We first need to study the topological nature of the space $\Lambda^{-1}(\lambda)$.

Let $\alpha:[a, b] \rightarrow R^{2}$ and $\beta:[c . d] \rightarrow R^{2}$ be parametrizations of two analytic arcs, where $\alpha$ and $\beta$ are injective, analytic functions. As above, consider the function

$$
\Lambda:[a, b] \times[c, d] \rightarrow R
$$

given by $\Lambda(x, y)=\|\alpha(x)-\alpha(y)\|$, for all $x, y \in S^{1}$.

We say that the $\operatorname{arcs} \alpha([a, b])$ and $\beta([c, d])$ are $\lambda$-parallel, if the curve $\{\alpha(t)+\lambda \eta(t) \mid$ $t \in[a, b]\}$ reparameterizes the curve $\beta([c, d])$, where $\eta(t)$ is the normal vector of the $\operatorname{arc} \alpha$ at the point $\alpha(t)$.

Lemma 2. Suppose that the analytic arcs $\alpha:[a, b] \rightarrow R^{2}$ and $\beta:[c . d] \rightarrow R^{2}$ are either $\lambda$-parallel or they do not contain parallel subarcs. Then $\Lambda^{-1}(\lambda)$ is homeomorphic to a space which is finite union of closed arcs.

Proof. A point $(x, y) \in[a, b] \times[c, d]$ is called a critical point if the derivative of $\Lambda$ at $(x, y)$ is 0 . It is easy to verify that a point $(x, y) \in[a, b] \times[c, d]$ is a critical point if and only if the chord $[\alpha(x), \beta(y)]$ is a binormal, that is, the tangent to the arc $\alpha$ at the point $\alpha(x)$ and the tangent to the $\operatorname{arc} \beta$ at the point $\beta(x)$ are orthogonal to the chord $[\alpha(x), \beta(y)]$. If in addition, the length of the chord $[\alpha(x), \beta(y)]$ is $\lambda$, that is 
$\Lambda(x, y)=\|\alpha(x)-\alpha(y)\|=\lambda$, then we say that $(x, y)$ is a $\lambda$-critical point and that the binormal chord $[\alpha(x), \beta(y)]$ is a $\lambda$-binormal. A point $(x, y) \in[a, b] \times[c, d]$ is called a $\lambda$-regular point if the derivative of $\Lambda$ at $(x, y)$ is not 0 and $\Lambda(x, y)=\lambda$.

Suppose first $\alpha$ and $\beta$ do not contain parallel subarcs. Then the set of $\lambda$-critical points is isolated, otherwise the analytic curve $\{\alpha(t)+\lambda \eta(t) \mid t \in[a, b]\}$, where $\eta(t)$ is the normal vector of the arc $\alpha$ at the point $\alpha(t)$, intersects $\beta([c, d])$ in an infinite set, which implies that the $\operatorname{arcs} \alpha$ and $\beta$ contain parallel subarcs. At the same time, the set of $\lambda$-regular points is a one-dimensional manifold. So $\Lambda^{-1}(\lambda)$ is a closed subset of $[a, b] \times[c, d]$ which is the union of a one-dimensional manifold and a finite collection of points, that is, $\Lambda^{-1}(\lambda)$ is homeomorphic to a space which is finite union of closed arcs.

Suppose now $\alpha$ and $\beta$ are $\lambda$-parallel arcs. Without loss of generality, $a=c, b=d$ and $\alpha(t)+\lambda \eta(t)=\beta(t)$, for every $t \in[a, b]$, where $\eta(t)$ is the normal vector of the $\operatorname{arc} \alpha$ at the point $\alpha(t)$. Then the set $B$ of all $\lambda$-critical points, which represents the $\lambda$-binormals, is homeomorphic to an arc. At the same time, the set of $\lambda$-regular points (if they exist) is a one-dimensional manifold $M$. So $\Lambda^{-1}(\lambda)$ is a closed subset of $[a, b] \times[c, d]$ which is the union of a one-dimensional manifold $M$ and the arc $B$. Consider the projection $\pi: M \cup B \rightarrow[a, b]$ given by $\pi(x, y)=x$. Note that $\pi \mid B: B \rightarrow[a, b]$ is a homeomorphism and for every $w \in[a, b]$, the set $\pi^{-1}(w)$ is a finite set, otherwise the analytic curve $\beta([a, b])$ intersects the circle with center $\alpha(w)$ and radius $\lambda$ in an infinite set. This implies that for every connected component $M^{\prime}$ of $M$, there are at most two points of accumulation of $M^{\prime}$ in $B$. That is, if two increasing sequences in $M^{\prime}$ converge to points of $B$, then they should converge to the same point, otherwise there should be point $w \in[a, b]$, with the property that $\pi^{-1}(w)$ is an infinite set. All this implies that $\Lambda^{-1}(\lambda)$ is homeomorphic to a space which is the finite union of closed arcs.

Theorem 2. Suppose $C$ is a piecewise analytic simple closed curve. If there is a car of length $\lambda$ which traverses the entire curve $C$ and $\lambda^{\prime}<\lambda$ then there is a car of length $\lambda^{\prime}$ which traverses $C$ entirely.

Proof. By the hypothesis, there exists a function $\psi: S^{1} \rightarrow \Lambda^{-1}(\lambda) \subset S^{1} \times S^{1}$ which represents the path of a car of length $\lambda$ along the entire curve. By Lemma $1, \psi$ is type $\pm(1,1)$, and therefore is homotopic to the diagonal function $\delta: S^{1} \rightarrow S^{1} \times S^{1}$. Let the homotopy be $H: S^{1} \times I \rightarrow S^{1} \times S^{1}$, that is $H(x, 1)=\psi(x)$ and $H(x, 0)= \pm(x, x)$ for all $x \in S^{1}$. In fact, since the curves $\psi$ and $\delta$ are parallel in the torus, we may assume that $H$ is injective. We note that $H\left(S^{1} \times\{1\}\right) \subset \Lambda^{-1}(\lambda)$ and $H\left(S^{1} \times\{0\}\right)=\Lambda^{-1}(0)$. Now let $\lambda^{\prime}<\lambda$ and let us consider $H^{-1}\left(\Lambda^{-1}\left(\lambda^{\prime}\right)\right) \subset S^{1} \times I$. It is clear that the closed set $H^{-1}\left(\Lambda^{-1}\left(\lambda^{\prime}\right)\right)$ separates $S^{1} \times\{1\}$ from $S^{1} \times\{0\}$ in $S^{1} \times I$. Furthermore, we may consider $C$ as the union of a finite collection of analytic $\operatorname{arcs}\left\{A_{1}, \ldots, A_{m}\right\}$ such that any two of them are either parallel or they do not contain parallel subarcs. By Lemma 2, $\Lambda^{-1}(\lambda)$ is homeomorphic to a space which is finite union of closed arcs and therefore, by elementary Alexander-duality arguments (see for example [4]), $H^{-1}\left(\Lambda^{-1}\left(\lambda^{\prime}\right)\right) \subset S^{1} \times I$ contains a cycle in $S^{1} \times I$ which separates $S^{1} \times\{1\}$ from $S^{1} \times\{0\}$. Let $\xi: S^{1} \rightarrow S^{1} \times I$ be a parameterization of this cycle, and note that $H \xi: S^{1} \rightarrow S^{1} \times S^{1}$ is type $\pm(1,1)$ and $H \xi\left(S^{1}\right) \subset \Lambda^{-1}\left(\lambda^{\prime}\right)$. Then by Lemma $1, H \xi: S^{1} \rightarrow \Lambda^{-1}\left(\lambda^{\prime}\right) \subset S^{1} \times S^{1}$ represents the path of a car of length $\lambda^{\prime}$ along the entire curve $C$. 


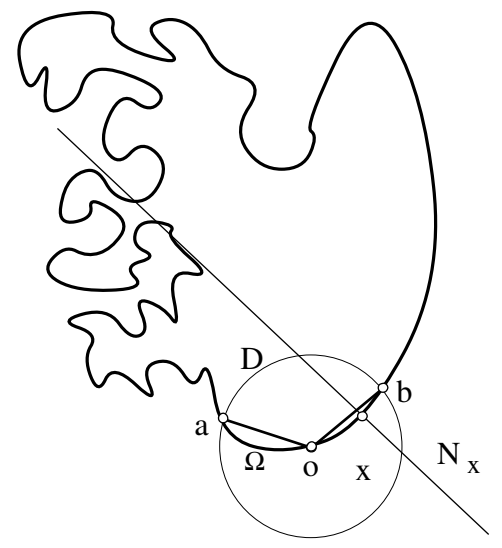

Fig. 4

To tackle Question 5, we need to define a $\lambda$-subarc. Recall that we wish to define it in terms of only a portion of the curve.

Definition 2. Let $C$ be a smooth curve. $\Omega \subset C$ is a $\lambda$-subarc of $C$ if there is a disc $D$ with center $0 \in C$ and radius $\lambda$, such that: (i) $\Omega=C \cap D$, (ii) $C \cap \partial D$ consists of precisely two points $\{a, b\}$, the endpoints of $\Omega$, (iii) if $0<\lambda^{\prime}<\lambda$ and $D^{\prime}$ is a disc with center 0 and radius $\lambda^{\prime}$, then $C \cap \partial D^{\prime}$ consists of precisely two points, and (iv) for all $x \in \Omega$, the normal $N_{x}$ to $C$ at $x$ is such that $N_{x} \cap D \cap C=\{x\}$.

In this definition, referring to Fig. 4 the hypotheses imply that a car $[b, 0]$ of length $\lambda$ moves along the curve $C$ within $D$ until it becomes $[0, a]$ such that both wheels move forward without stopping or backing up. This follows immediately from the following lemma, which says that if the back wheel is forced to move in the opposite direction in order to enable the car to keep moving forward, then the curve $C$ is perpendicular to the car at the back wheel. Note also that if $\Omega$ is a $\lambda$-subarc and $\lambda^{\prime}$ and $D^{\prime}$ are as in Definition 2, then $\Omega \cap D^{\prime}$ is a $\lambda^{\prime}$-subarc.

Lemma 3. Let $\vartheta=\left(\vartheta_{1}, \vartheta_{2}\right):(-\varepsilon, \varepsilon) \rightarrow \Lambda^{-1}(\lambda)$ be a smooth function such that $(d \vartheta / d t)(0) \neq 0$. If $\left(d \alpha \vartheta_{1} / d t\right)(0)=0$, then the tangent to $C$ at the point $\alpha(y)$ is perpendicular to the line passing through $\alpha(x)$ and $\alpha(y)$ where $\vartheta(0)=(x, y)$.

Proof. For $t \in(-\varepsilon, \varepsilon)$, let $\theta(t)$ be the angle of the unit vector in the direction of $\alpha \vartheta_{1}(t)-\alpha \vartheta_{2}(t)$. So $\theta:(-\varepsilon, \varepsilon) \rightarrow R$ is a smooth function such that

$$
\alpha \vartheta_{1}(t)+\lambda(\cos \theta(t), \sin \theta(t))=\alpha \vartheta_{2}(t)
$$

Differentiating, and assuming that $(d \vartheta / d t)(0) \neq 0$ and $\left(d \alpha \vartheta_{1} / d t\right)(0)=0$, we have that $\left(d \alpha \vartheta_{2} / d t\right)(0)=\left(d \vartheta_{2} / d t\right)(0)(d \alpha / d t)\left(\vartheta_{2}(0)\right)$ is parallel to $(-\sin \theta(0), \cos \theta(0))$ and therefore perpendicular to the segment $\lambda(\cos \theta(0), \sin \theta(0))=\alpha\left(\vartheta_{2}(0)\right)-\alpha\left(\vartheta_{1}(0)\right)$, 
which implies that at the point $\alpha(x)$, the curve $C$ is perpendicular to the line that passes through $\alpha(x)$ and $\alpha(y)$.

At this point we need some elementary notions from Morse Theory and Degree Theory which we will use below. See [2] for example.

If $C$ is a smooth curve, then the function

$$
\Lambda: S^{1} \times S^{1} \rightarrow R
$$

given by $\Lambda(x, y)=\|\alpha(x)-\alpha(y)\|$, for all $x, y \in S^{1}$, is a smooth function. It is easy to see that the critical points of $\Lambda$ (the points $(x, y) \in S^{1} \times S^{1}$ in which the derivative of $\Lambda$ is zero) are the points of the diagonal $\Delta$ and the points in which the tangents to $C$ at $\alpha(x)$ and $\alpha(y)$ are both perpendicular to the line through $\alpha(x)$ and $\alpha(y)$. Then $\lambda \in R$ is a critical value of $\Lambda$ if $\Lambda(x, y)=\lambda$ for $(x, y)$ a critical point of $\Lambda$. If $\lambda \in R$ is not a critical value of $\Lambda$, then $\lambda$ is called a regular value and $\Lambda^{-1}(\lambda)$ is a finite collection of cycles $\Sigma_{1}, \ldots, \Sigma_{\rho}$. Moreover, if the interval $\left[\lambda^{\prime}, \lambda\right]$ contains only regular values, then $\Lambda^{-1}\left(\left[\lambda^{\prime}, \lambda\right]\right)$ is homeomorphic to the disjoint union $\Sigma_{i} \times\left[\lambda^{\prime}, \lambda\right]$, since the behavior of the function $\Lambda$ changes only at the critical values.

Finally, we recall that if $f: S^{1} \rightarrow S^{1}$ is a smooth function, then the degree of $f$ can be calculated on a regular point $x$ of the image in the following way. As $f^{-1}(x)=$ $\left\{a_{1}, \ldots, a_{\tau}\right\}$ consists of a finite set of points, then each point $a_{i}$ contributes a +1 or a -1 depending on whether the function $f$ preserves or does not preserve orientation locally around $a_{i}$. The degree of $f$ is the sum of all these +1 's and -1 's.

The following theorem shows that the problem of traversing the entire curve with a car of length $\lambda$ is not a local problem, that is, using just information that comes from a piece of the curve $C$, it is impossible to conclude that a car of length $\lambda$ cannot traverse the entire curve $C$.

Theorem 3. If $C$ is a simple smooth curve which contains a $\lambda$-subarc, then there is $\lambda^{\prime}<\lambda$, sufficiently close to $\lambda$, and a car of length $\lambda^{\prime}$ which traverses the entire curve $C$. If in addition, $C$ is a simple analytic curve, then for every $\lambda^{\prime}<\lambda$, there is a car of length $\lambda^{\prime}$ which traverses the entire curve $C$.

Proof. We begin by noting that without loss of generality $\lambda$ can be assumed to be a regular value of $\Lambda$. Thus $\Lambda^{-1}(\lambda)$ consists of a disjoint set of cycles $\Sigma_{1}, \ldots, \Sigma_{\rho}$, each of which is in $S^{1} \times S^{1}-\Delta$. That is, the type of $\Sigma_{i} \subset S^{1} \times S^{1}$ is either $\pm(1,1)$ or $(0,0)$. If some of the cycles $\Sigma_{i}$ have type $\pm(1,1)$, then by Lemma 1 , the theorem is proved.

Since $C$ contains a $\lambda$-subarc, one can choose $0, a, b, D$ and $\Omega$ so that (i)-(iv) of Definition 2 hold.

Consider $\left(\alpha^{-1}(0), \alpha^{-1}(b)\right)$ in $\Delta^{-1}(\lambda)$ and suppose that $\left(\alpha^{-1}(0), \alpha^{-1}(b)\right) \in \Sigma_{j}$, for some $j=1, \ldots, \rho$. Let $\psi=\left(\psi_{1}, \psi_{2}\right): S^{1} \rightarrow \Sigma_{j} \subset \Delta^{-1}(\lambda) \subset S^{1} \times S^{1}$ be a parameterization. It will be enough to prove that the degree of $\psi_{1}$ is \pm 1 . By Lemma 1, $\alpha^{-1}(0)$ is a regular value of $\psi_{1}$, then we can calculate the degree of $\psi_{1}$ by looking at $\psi_{1}^{-1}\left(\alpha^{-1}(0)\right)$. Since by (ii), $C \cap \partial D=\{a, b\}$, either $\psi_{1}^{-1}\left(\alpha^{-1}(0)\right)$ consists of a single point, that is, $\psi_{1}^{-1}\left(\alpha^{-1}(0)\right)=\left\{\left(\alpha^{-1}(0), \alpha^{-1}(b)\right)\right\}$ and therefore the degree of $\psi_{1}$ is \pm 1 , or $\psi_{1}^{-1}\left(\alpha^{-1}(0)\right)=\left\{\left(\alpha^{-1}(0), \alpha^{-1}(b)\right),\left(\alpha^{-1}(0), \alpha^{-1}(a)\right)\right\}$. If this is the case, Lemma 1 
implies that the car $[0, b]$ moves along the curve $C$ within $D$ until it becomes the car $[a, 0]$ in such a way that both wheels move forward without stopping or backing up. Similarly, the car $[0, a]$ moves backwards along the curve $C$ within $D$ until it becomes the car $[b, 0]$ in such a way that both wheels move backward without stopping. This implies that if $(x, y) \in \Sigma_{j}$, then $(y, x) \in \Sigma_{j}$. We prove next that this implies that the type of $\Sigma_{j}$ is $\pm(1,1)$. If we identify the point $(x, y)$ with $(y, x)$ in $S^{1} \times S^{1}-\Delta$ we obtain an open mobius band $M$ and the quotient map $\pi: S^{1} \times S^{1}-\Delta \rightarrow M$ is a double cover. Suppose the type of $\Sigma_{j}$ is not $\pm(1,1)$, then $\Sigma_{j}$ has type $(0,0)$ and consequently $\Sigma_{j}$ is null homotopic in $S^{1} \times S^{1}-\Delta$, which implies that $\pi\left(\Sigma_{j}\right)$ is null homotopy in $M$ and therefore $\pi^{-1}\left(\pi\left(\Sigma_{j}\right)\right)$ has two components, which is a contradiction to the fact that $(x, y) \in \Sigma_{j}$ if and only if $(y, x) \in \Sigma_{j}$. The theorem now follows easily from Theorem 2.

\section{4. "Model Trains"}

Definition 3. A model train with $n$ cars of lengths $\lambda_{1}, \ldots, \lambda_{n}$ running along the track described by $C$ consists of $n+1$ points $\left\{a_{1}, \ldots, a_{n+1}\right\} \subset C$ such that

(i) for $i=1, \ldots, n,\left\|a_{i}-a_{i+1}\right\|=\lambda_{i}$,

(ii) for $i=2, \ldots, n$, the points $a_{i+1}, a_{i}, a_{i-1}$ orient the curve $C$ positively.

Note that according to our definition the cars of a model train can intersect each other and if $\lambda_{i}=\lambda_{i+1}$ the $i$ th and the $(i+1)$ th cars can be in the same position.

We also say that there exists a model train with $n$ cars of lengths $\lambda_{1}, \ldots, \lambda_{n}$ which traverses the curve $C$ entirely if there exists a function

$$
\Psi: S^{1} \rightarrow S^{1} \times \cdots \times S^{1}
$$

such that for all $x \in S^{1},\left\{\alpha\left(\Psi_{1}(x)\right), \ldots, \alpha\left(\Psi_{n+1}(x)\right)\right\}$ is a train running on $C$ with $n$ cars having lengths $\lambda_{1}, \ldots, \lambda_{n}$ and

$$
\Psi_{1}: S^{1} \rightarrow S^{1}
$$

is a continuous function with degree \pm 1 , where, of course, $\Psi=\left(\Psi_{1}, \ldots, \Psi_{n+1}\right)$.

We note that in this case the projection of $\Psi$ on the first two coordinates represents the path of a car of length $\lambda_{1}$ which traverses $C$ entirely. Therefore $\Psi_{2}$, the projection of $\Psi$ on the second coordinate, is a function of degree \pm 1 . Proceeding inductively, we can verify that the projection of $\Psi$ on coordinates $i, i+1$ gives rise to the path of a car of length $\lambda_{i}$ which traverses $C$ entirely; therefore $\Psi(i+1)$ is a function of degree \pm 1 , $i=0, \ldots, n$.

We will now prove the following theorem.

Theorem 4. Suppose that a car of length $\lambda_{1}$ traverses the entire piecewise analytic curve curve $C$. Then, for any integer $n \geq 1$ and $\lambda_{1} \geq \lambda_{2} \geq \cdots \geq \lambda_{n}$ there exists a train with $n$ cars having lengths $\lambda_{1}, \ldots, \lambda_{n}$ which traverses the entire curve $C$. 
Proof. We begin by proving the theorem for two-car trains. If $\lambda_{1}=\lambda_{2}$ then both cars can be in the same position, just in opposite direction, so we may assume $\lambda_{1}>\lambda_{2}$. By Lemma 1 and Theorem 2 , let $\varphi=\left(\varphi_{1}, \varphi_{2}\right): S^{1} \rightarrow S^{1} \times S^{1}$ be a function of type $\pm(1,1)$ which represents the path of a car of length $\lambda_{1}$. Let

$$
E: S^{1} \times S^{1} \rightarrow R
$$

be the function defined by $E(x, y)=\left\|\alpha \varphi_{2}(x)-\alpha(y)\right\|$.

Then $E^{-1}\left(\lambda_{2}\right)$ is the space of all possible positions on the curve $C$ of a two-car train with car lengths $\lambda_{1}$, prescribed by $\varphi$, and car lengths $\lambda_{2}$. Intuitively we must think of a point of $S^{1} \times S^{1}$ as a pair of points of $S^{1}$, where the first coordinate represents a car length $\lambda_{1}$, prescribed by $\varphi$, and the second coordinate represents a point of the curve $C$.

Let us consider the curves $\xi_{i}=\left\{\left(x, \varphi_{i}(x)\right) \in S^{1} \times S^{1} \mid x \in S^{1}\right\}, i=1$, 2. Clearly, $\xi_{2} \subset E^{-1}(0)$ and $\xi_{1} \subset E^{-1}\left(\lambda_{1}\right)$ are two cycles of type $\pm(1,1)$ in $S^{1} \times S^{1}$ which do not intersect. In fact, $\Gamma=\left\{(x, y) \in S^{1} \times S^{1} \mid\right.$ the points $\alpha \varphi_{1}(x), \alpha \varphi_{2}(x), \alpha(y)$ orient the curve $C$ positively\} is a band in $S^{1} \times S^{1}$, homeomorphic to $S^{1} \times\left[0, \lambda_{1}\right]$, whose boundary is $\xi_{1}$ and $\xi_{2}$. If $\lambda_{2}<\lambda_{1}$, since $\xi_{2} \subset E^{-1}(0)$ and $\xi_{1} \subset E^{-1}\left(\lambda_{1}\right)$, then $E^{-1}\left(\lambda_{2}\right)$ separates $\xi_{1}$ from $\xi_{2}$ in $\Gamma$. Using the ideas of Lemma 2, as in the proof of Theorem 2, and by elementary Alexander-duality arguments (see [4]), we have that $E^{-1}\left(\lambda_{2}\right) \subset \Gamma$ contains a cycle $\xi_{3}$ which separates $\xi_{1}$ from $\xi_{2}$ in $\Gamma$ and is therefore of type \pm 1 .

Let $\psi: S^{1} \rightarrow S^{1} \times S^{1}$ be a parameterization of $\xi_{3}$. We first note that $\psi=\left(\psi_{1}, \psi_{2}\right)$ is a curve of type $\pm(1,1)$ in $S^{1} \times S^{1}$ with the property $\psi\left(S^{1}\right) \subset E^{-1}\left(\lambda_{2}\right) \subset \Gamma$. This implies that the function $\Psi: S^{1} \rightarrow S^{1} \times S^{1} \times S^{1}$, given by the coordinate functions $\left(\varphi_{1} \psi_{1}, \varphi_{2} \psi_{1}, \psi_{2}\right)$ represents the path of a train with two cars of lengths $\lambda_{1}, \lambda_{2}$ along the entire curve $C$.

For trains with three cars, we must think now of a point of $S^{1} \times S^{1}$ as a pair of points of $S^{1}$, where the first coordinate represents a train of two cars, prescribed by $\Psi=\left(\Psi_{1}, \Psi_{2}, \Psi_{3}\right)$, and the second coordinate represents a point of the curve $C$. Now $E: S^{1} \times S^{1} \rightarrow R$ is given by $E(x, y)=\left\|\alpha \Psi_{3}(x)-\alpha(y)\right\|$. Proceeding in the same way, we can prove the theorem for trains with three cars and using induction we can prove the theorem for trains of any number of cars.

Corollary 1. If $C$ is a simple analytic closed curve which contains a $\lambda$-subarc, then there exists for all $n \geq 1$ and $\lambda>\lambda_{1} \geq \cdots \geq \lambda_{n}$ a train with $n$ cars of lengths $\lambda_{1}, \ldots, \lambda_{n}$ which traverses the entire curve $C$.

Proof. The proof follows immediately from Theorems 3 and 4 .

\section{Distance Traveled}

Consider a polygonal $P$ formed by two segments $l_{1}, l_{2}$ of lengths 3 and 4 , respectively, and $2 n-1$ short and $2 n$ long segments of lengths $1+\varepsilon$ and 1 , and 3 and $3+\varepsilon$, respectively, as shown in Fig. 5(a) for $n=4$. Suppose that we want to move a car $R$ of length 1.5 (represented by the line segment with endpoints labeled $b$ and $f$ in Fig. 5(a)) from $l_{1}$ to 


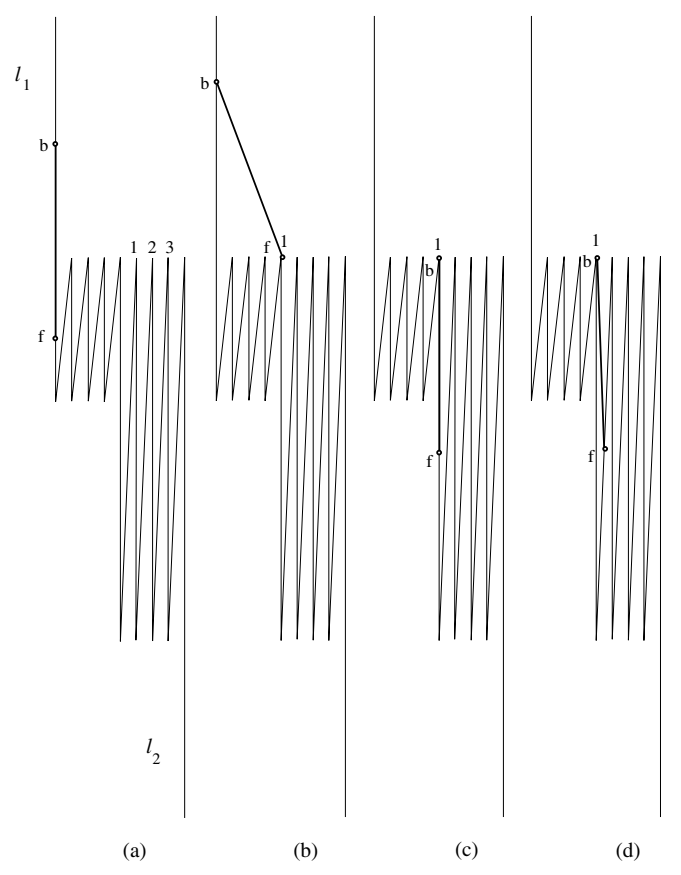

Fig. 5

$l_{2}$. It is clear that to achieve our goal $R$ must first pass through a position in which $b$ is on $l_{1}$ and $f$ is on the point labeled 1, Fig. 5(b). Then $R$ must move to a position in which $f$ lies on point 2, Fig. 6(a). However, to achieve this, $R$ must pass through the positions shown in Figs. 5(c) and (d), and then move to the position shown in Fig. 6(a). A similar process has to be followed to move $f$ to point $3,4, \ldots$ In each of our iterations, $b$ must move from a point on $l_{1}$ to point 1 and back to $l_{1}$. Since this has to be done $n$ times, it follows that the distance traveled by $b$, and hence $f$, is quadratic. Since the length of $P$ is $8 n+6+8 \varepsilon$, it follows that the distance traveled by the wheels of $R$ can be arbitrarily large compared with the length of $P$.

In a similar way we can see that if instead of a car we have a train $T$ with two cars (i.e. two segments of length 1.5 joined at one of their ends, making $l_{1}$ and $l_{2}$ longer to allow $T$ to move), to move $T$ from $l_{1}$ to $l_{2}$, the wheels of $T$ must travel a distance proportional to $n^{3}$. For trains with $k$ cars, we can easily see that the distance traveled by their wheels is $O\left(n^{k+1}\right)$ (for each car we must repeat the same procedure that we did for $R$ ).

By completing $P$ to a simple closed polygon we obtain:

Theorem 5. Let $C$ be a simple closed curve and let $B$ be a car that can traverse $C$. Then the distance traversed by the wheels of $R$ in a complete traversal of $C$ can be arbitrarily large with respect to the length of $C$. Moreover, if $C$ is a polygon with $n$ vertices, and has length $O(n)$, to complete a whole traversal of $C$ the wheels of a train with $k$ cars may have to travel a distance of $O\left(n^{k+1}\right)$. 


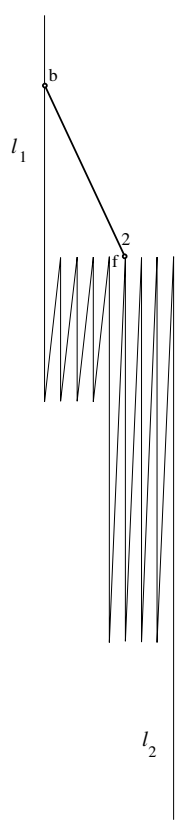

(a)

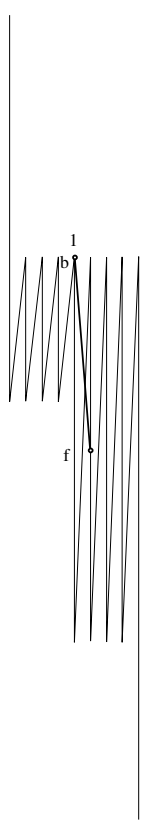

(b)

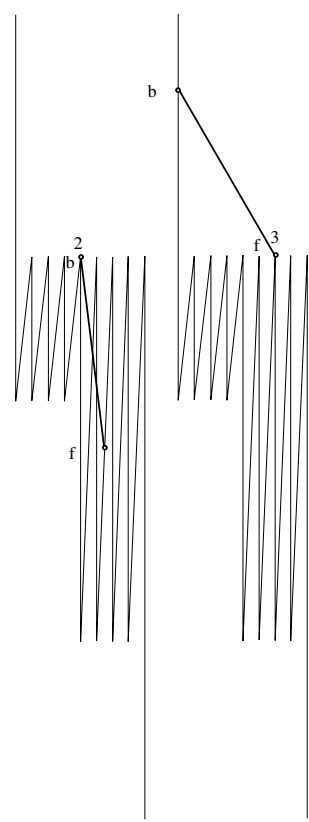

(c)

(d)

Fig. 6

\section{Acknowledgement}

The authors thank the referee for useful comments concerning the proof of Theorem 2 .

\section{References}

1. J.E. Goodman, J. Pach, and C.K. Yap, Mountain climbing, ladder moving, and the ring-width of a polygon, American Mathematical Monthly, 96(6), 494-510, 1989.

2. J. Milnor, Differential Topology, Lectures on Modern Mathematics, Vol. 2, T. Saaty, ed., Wiley, New York, 1964.

3. D. Rolfsen, Knots and Links, Mathematics Lecture Series No. 7, Publish or Perish, Houston, TX, 1976.

4. E. H. Spanier, Algebraic Topology, Springer-Verlag, New York, 1966.

Received June 17, 2004, and in revised form September 25, 2005, September 6, 2006, and January 17, 2007. Online publication May 18, 2007. 